\title{
Recurrence of depression in those with prepubertal onset was associated with higher rates of depression in relatives
}

Wickramaratne PJ, Greenwald S, Weissman MM. Psychiatric disorders in the relatives of probands with prepubertal-onset or adolescent-onset major depression.J Am Acad Child Adolesc Psychiatry 2000 Nov;39:1396-405.

QUESTION: Is the pattern of recurrence and stability of depression into adulthood different as a function of prepubertal and adolescent onset and familial loading?

\section{Design}

Inception cohort followed up for 10-15 years with family study of first degree relatives.

\section{Setting}

Department of Psychiatry, Columbia University, College of Physicians and Surgeons, New York, USA.

\section{Patients}

83 prepubertal onset depressed probands (mean age first depression 9 y, 91\% followed into adulthood), 73 adolescent onset depressed probands (mean age first depression 14 y, 81\% followed into adulthood), and 120 control probands (91 prepubertal and 37 adolescents, $65 \%$ followed into adulthood) participated. Pubertal status of the participants was determined at the initial assessment by Tanner staging.

\section{Assessment of prognostic factors}

The psychiatric status of first degree family members was obtained using the Schedule for Affective Disorders and Schizophrenia Lifetime Version Modified for the Study of Anxiety Disorders. Family history information was obtained from multiple informants for those who could not be interviewed. Final psychiatric diagnosis of family members was determined by an experienced psychiatrist or psychologist after a review of all available information.

\section{Main outcome measures}

Recurrence and continuity of depression into adulthood.

Sources of funding:

National Institute of Mental Health;

National Alliance for

Research in

Schizophrenia and

Depression Senior

Investigator's award:

John D and Catherine

TMacArthur

Foundation.

For correspondence: Dr PJ Wickramaratne, Department of

Psychiatry, College of Physicians and Surgeons, Columbia University, New York State Psychiatric Institute, 1051 Riverside Drive, Unit 24, New York, $N Y$ 10032, USA. Fax +1 2125683534.

\section{Main results}

25 prepubertal onset depressed probands (38\%) and 30 adolescent onset depressed probands $(51 \%)$ had a recurrence during follow up. Recurrence of prepubertal onset depression was associated with higher rates of depression in relatives (odds ratio [OR] 3.5, 95\% CI 1.5 to 7.8). The same association was not found for recurrence of adolescent onset depression (OR 1.4, CI 0.7 to 2.7). No association was found between a family history of depression and continuity of depression into adulthood (adulthood defined as $>18$ y of age) for either prepubertal or adolescent onset depression (OR 3.1, CI 0.9 to 11.2 and OR 1.2, CI 0.6 to 2.4, respectively).

\section{Conclusions}

Recurrence of prepubertal onset depression was associated with higher rates of depression in relatives. Recurrence was not a function of family loading for those with adolescent onset.

\section{COMMENTARY}

There has been ample debate surrounding the classification of depression as either a single disease characterised by a continuum of symptoms, or conversely, as a subset of distinct diseases with unique clinical characteristics and course. ${ }^{12}$ Recent evidence has begun to converge in support of a clinically homogenous condition in which symptom manifestations within individuals are readily changeable and expressed longitudinally both above and below the accepted diagnostic threshold. ${ }^{3}$ The present investigation by Wickramaratne $e t$ al takes this debate one step further by asking whether patients who present clinically with relatively homogenous symptoms may indeed signify diverse subgroups based on a family history of major depressive disorder (MDD). Although some specific associations were identified, the similarities among groups are arguably the more notable findings. For example, relatives of both prepubertal and adolescent onset patients had significantly higher rates of MDD than never depressed control subjects. Furthermore, no difference in rates of MDD or other psychiatric disorders was found among first degree relatives of prepubertal compared with adolescent onset patients. Taken together, this supports the hypothesis that MDD is a homogenous condition, familial in nature, and not readily discernable by timing of development.

The addition of more fine grained analyses revealed that MDD recurrence in prepubertal onset patients (but not adolescent onset patients) is associated with a family history of MDD. This points to some prognostic value in the systematic evaluation of a patient's family history. However, the meaningfulness of this information in terms of treatment selection, or the likeliness of treatment adherence, remains an important area of inquiry.

Overall, this investigation represents a substantial contribution to our continued pursuit of more accurate classification of MDD. The prospective nature of this study, the broad time span encompassed by the longitudinal assessments, and the inclusion of the family study design elements, make this a particularly appropriate model for continued work in this area.

\section{Lisa Dierker, $\mathrm{PhD}$ Wesleyan University Middletown, Connecticut, USA}

1 Winokur G. All roads lead to depression: clinically homogeneous, etiologically heterogeneous. J Affect Disord 1997;45:97-108.

2 Kendler KS, Gardner CO Jr. Boundaries of major depression: an evaluation of DSM-IV criteria. Am J Psychiatry 1998; 155:172-7.

3 Judd LL, Akiskal HS, Maser JD, et al. A prospective 12-year study of subsyndromal and syndromal depressive symptoms in unipolar major depressive disorders. Arch Gen Psychiatry 1998;55:694-700. 